\title{
Serum and local IL-4, IL-5, IL-13 and immunoglobulin E in allergic rhinitis
}

\author{
Atanas Nachev Vlaykov ${ }^{1,2}$, Tanya Tacheva Tacheva ${ }^{3}$, Tatyana Ivanova Vlaykova ${ }^{3}$, Valentin Kostov Stoyanov ${ }^{1}$ \\ 1Department of Otorhinolaryngology and Ophthalmology, Medical Faculty, Trakia University, Stara Zagora, Bulgaria \\ 2Department of Otorhinolaryngology, Ophthalmology and Maxillofacial Surgery, Medical University, Pleven, Bulgaria \\ ${ }^{3}$ Department of Chemistry and Biochemistry, Medical Faculty, Trakia University, Stara Zagora, Bulgaria \\ Adv Dermatol Allergol 2020; XXXVII (5): 719-724 \\ DOI: https://doi.org/10.5114/ada.2020.100483
}

\begin{abstract}
Introduction: Allergic rhinosinusitis (AR) is a clinical manifestation of a type 1 hypersensitive reaction. A complex of reactions involving components of the immune system - cells, mediators, cytokines, neuropeptides, adhesion molecules etc., are involved in the manifestation of the disease symptoms.

Aim: To evaluate the role of some serum and local cytokines and IgE molecules in the pathogenesis of AR comparing results in patients and healthy controls.

Material and methods: The study was conducted at the Prof. Dr. St. Kirkovich University Hospital and Medical University, Stara Zagora, Bulgaria.

Results: A trend towards higher serum levels in patients with AR compared to controls was found for IL-4, but with no significant difference. In the group of AR patients, those with the intermittent form had higher, although with no significance, interleukin 4 (IL-4) levels in the lavage compared to those with the persistent form. In nasal lavage fluids a tendency towards higher IL-5 levels was found in intermittent AR patients compared to those with persistent AR. A slight trend towards significantly higher serum levels of IL-13 in overweight patients compared to those with normal weight was found.

Conclusions: Regardless of the obvious differences of the concentrations of the cytokines studied in our groups, oftentimes no significant difference is observed. More studies should be conducted in order to show the role of $\mathrm{IL}-4,-5,-13$, and IgE in the pathogenesis and severity of the disease.
\end{abstract}

Key words: allergic rhinitis, nasal lavage, interleukin, immunoglobulin E, cytokines.

\section{Introduction}

Rhinosinusitis is characterized by inflammation of nasal and mucous membranes of sinuses, manifested by the following symptoms: anterior or posterior rhinorrhoea, sneezing, difficult nasal breathing and/or itching and burning inside the nose. These symptoms occur during two or more consecutive days for more than $1 \mathrm{~h}$ for most of the days [1].

Allergic rhinosinusitis is a symptomatic nasal disease provoked by exposure of nasal mucosa to allergens, resulting in IgE-mediated inflammation [2].

Atopic diseases such as allergic rhinitis, bronchial asthma, drug allergy etc. are characterized by lgE-mediated immune response against foreign antigens - allergens. Genetic predisposition, combined with environmental factors, contributes to the development of the pathol- ogy. These IgE-mediated allergic reactions are most commonly found in mucous membranes (nose, conjunctiva, airways, gastrointestinal tract) or skin surfaces, where high levels of mast cells covered with IgE molecules are found [3].

Allergic rhinosinusitis is a clinical manifestation of a type 1 hypersensitive reaction. A complex of reactions involving components of the immune system - cells, mediators, cytokines, neuropeptides, adhesion molecules etc., are involved in manifestation of the disease symptoms [4].

After exposure to allergens, they are phagocytized by antigen-presenting cells (APCS): macrophages, dendritic cells or Langerhans cells, located in the epithelium of the respiratory mucosa. After processing, antigens are expressed on cell surface associated with the major his-

Address for correspondence: Assist. Prof. Atanas Nachev Vlaykov, Department of Otorhinolaryngology and Ophthalmology, Medical Faculty, Trakia University, Stara Zagora, Bulgaria, phone: +359886072199, e-mail: at.vlaykov@gmail.com Received: 19.02.2019, accepted: 11.03.2019. 
tocompatibility complex (MHC) molecules. At the same time, the activated APCs carry out synthesis of early regulatory cytokine molecules IL-25 and IL-33 [5]. This process is followed by activation of other CD4 + (Th-Ly) cells involved in the immune response, particularly Th2 subclass lymphocytes. It is believed that once activated, Th2 start secreting specific cytokines: IL-3, IL-4, IL-5, IL-13, etc. which, through a series of complex cell interactions, lead to increased synthesis of eosinophils and transformation of B-lymphocytes into antibody secretory cells plasmatic cells.

In the course of an already started allergic reaction, plasmatic cells produce IgE antibodies against a particular allergen. Once formed and released in the bloodstream, IgE molecules bind with high affinity to mast cell receptors localized in the skin, nose and respiratory tract mucosa, providing an allergen-specific receptor available for future interaction with allergens [6].

Mechanisms regulating IgE synthesis are different compared with other classes, and its levels gradually increase in infancy to reach adult values at about 15-20 years of age. These IgE values are usually low, and an IgE specific allergen response is often followed by a significant increase in their total serum concentration. It is important to note that commonly lower serum IgE-total levels in healthy individuals do not entirely exclude the presence of a greater amount of antigen-specific antibodies and development of a hypersensitivity reaction after interaction with corresponding foreign molecules [7].

\section{Aim}

The aim of the study was to evaluate the role of some serum and local cytokines and lgE molecules in the pathogenesis of AR comparing results in patients and healthy controls. Additionally, the study aimed to compare the differences between the two main clinical forms of $A R$ - intermittent $A R(I A R)$ and persistent $A R$ (PAR).

\section{Material and methods}

\section{Patients and controls}

The study was conducted at the Prof. Dr. St. Kirkovich University Hospital and Medical University, Stara Zagora, Bulgaria. During the survey from January to December 2016, 139 participants (111 with allergic rhinitis and 28 controls) were enrolled. In the group of patients, 54 (48.65\%) had IAR and 57 (51.35\%) PAR. The mean age in patients with AR was $45.59 \pm 16.37$ (22-84) and in the control group: $42.96 \pm 15.06$ (19-63). The patients group consisted of 72 (53.7\%) women and 62 (46.3\%) men. Gender distribution among controls was 18 (62.1\%) women and 11 (37.9\%) men. Patients with both forms of AR did not take any antiallergic, anti-histamine, topical or systemic corticosteroid drugs, and nonsteroidal anti-inflammatory drugs (NSAIDs), at least a month before the study.
The control group participants were strictly selected all individuals were healthy, without acute or chronic inflammatory process that could affect immune response. Persons with allergic family history were excluded from this group.

\section{Collection of lavage fluid and serum}

The collection of lavage fluid was performed by washing the nasal cavity with $6 \mathrm{ml}$ of saline, tempered to body temperature, a total of 30 times. All the lavages were centrifuged at $4^{\circ} \mathrm{C}$ for $10 \mathrm{~min}$ at $500 \mathrm{~g}$ as both sediment and supernatant were stored at $-20^{\circ} \mathrm{C}$. Venous blood was collected in tubes with Na2EDTA as an anticoagulant. The samples were centrifuged, separated and the serum was kept at $-20^{\circ} \mathrm{C}$ until the assay.

\section{Measurement of ILs and IgE levels}

$\mathrm{IL}$ and IgE levels were measured in serum and nasal lavage with enzyme-linked immunosorbent assay (ELISA) - Invitrogen ELISA kit. The results were expressed as optical density (OD) at $450 \mathrm{~nm}$ and calculated according to the OD of the standard.

\section{Ethical aspects}

All participants, after detailed presentation of the aims, tasks and methodology of the study and the opportunity for discussion, signed an informed consent form. The form was prepared in accordance with principles of the Helsinki Declaration for Good Clinical and Laboratory Practice and approved on 07.05.2014 at a meeting of the Medical Ethics Committee at the Medical University/University Hospital, Stara Zagora.

\section{Statistical analysis}

Statistical analyses were performed using SPSS 16.0 for Windows (SPSS Inc.). The continuous variables with normal distribution were compared between 2 or more independent groups by Student $t$-test or One-way ANOVA test with LSD Post hoc analysis, while those variables with non-normal distribution were compared by using Mann-Whitney $U$ test or Kruskal-Wallis $H$ test. The non-paramedic Spearman rank correlation test and linear correlation analysis were used in determination of correlation between the quantitative variables. The results were evaluated at a 95\% confidence interval, and significance was assessed at $p<0.05$.

\section{Results}

After statistical analyses of the results we found higher serum IgE levels in patients with AR compared to control individuals however, without significant difference ( $237.84 \pm 38.71 \mathrm{U} / \mathrm{ml}$ vs. $175.44 \pm 79.89 \mathrm{U} / \mathrm{ml}, p=0.474)$.

A similar trend for higher serum levels in patients with AR compared to controls, but with no significant 
difference was found for IL-4 (29.48 $\pm 17.77 \mathrm{pg} / \mathrm{ml}$ vs. 7.88 $\pm 4.70 \mathrm{pg} / \mathrm{ml}, p=0.188$ ) (Figure 1). When measuring the levels of IL-4 in the lavage of AR patients and control individuals we found no differences ( $3.02 \pm 0.44$ vs. $2.31 \pm 0.12$ $\mathrm{pg} / \mathrm{ml}, p=0.668)$. In the group of AR patients, those with the intermittent form had higher, although with no significance, IL-4 levels in the lavage compared to those with the persistent form $(3.65 \pm 0.67 \mathrm{pg} / \mathrm{ml}$ vs. $2.39 \pm 0.2 \mathrm{pg} / \mathrm{ml}$, $p=0.651)$.

In IL-5 serum levels we did not find any statistical differences between AR patients and control subjects ( $6.31 \pm 1.24$ $\mathrm{pg} / \mathrm{ml}$ vs. $5.61 \pm 2.2 \mathrm{pg} / \mathrm{ml}, p=0.842$ ). On the other hand, in the lavage a tendency towards higher IL-5 levels in IAR patients compared to those with PAR was found (13.43 \pm 4.77 $\mathrm{pg} / \mathrm{ml}$ vs. $3.84 \pm 1.81 \mathrm{pg} / \mathrm{ml}, p=0.088$ ) (Figure 2).

Control individuals had lower IL-13 levels in the serum $(5.04 \pm 3.75 \mathrm{pg} / \mathrm{ml})$ compared to the patients with IAR $(15.05 \pm 5.77 \mathrm{pg} / \mathrm{ml}, p=0.296)$ and PAR $(16.76 \pm 7.4$ $\mathrm{pg} / \mathrm{ml}, p=0.433)$, although with no significant difference.

According to body mass index (BMI), a weak trend for higher IL-4 serum levels in overweight patients (61.80 $\pm 40.0 \mathrm{pg} / \mathrm{ml}$ ) compared to those with normal weight (2.81 $\pm 0.58 \mathrm{pg} / \mathrm{ml}, p=0.160)$ and underweight (10.30 $\pm 5.59 \mathrm{pg} / \mathrm{ml}, p=0.220$ ) appeared (Figure 3). A slight trend

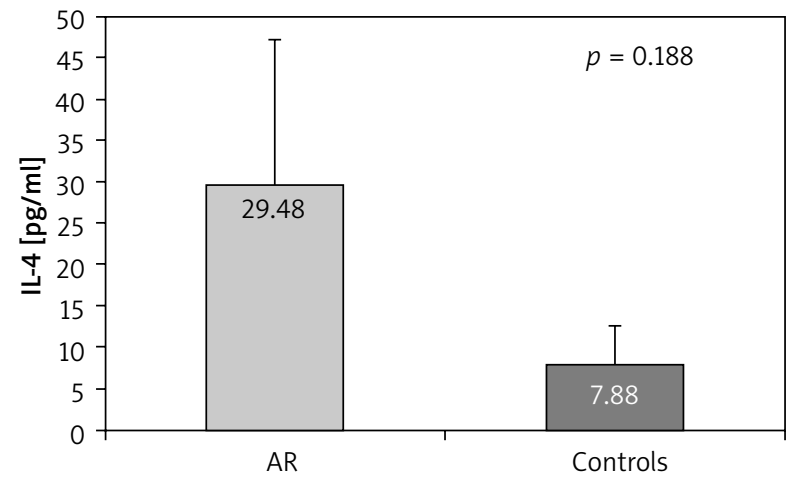

Figure 1. IL-4 levels in the serum of AR patients and controls

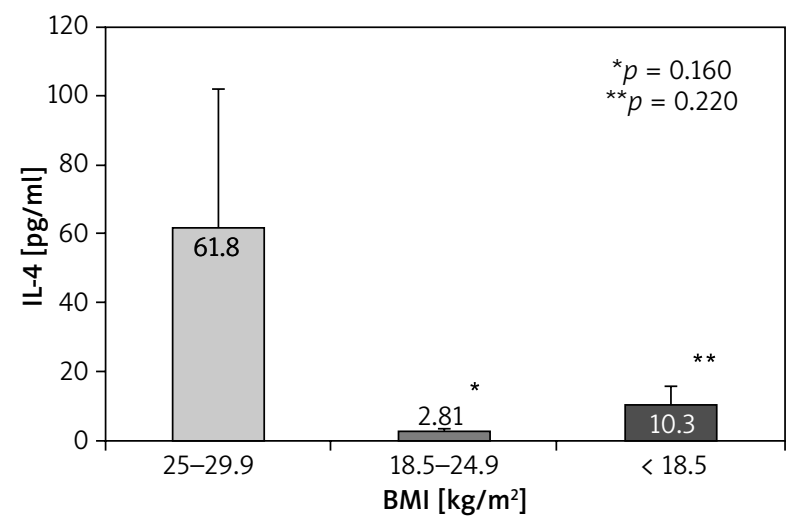

Figure 3. IL-4 serum levels in AR patients according to BMI towards significantly higher serum levels of IL-13 in overweight patients compared to those with normal weight was found $(24.99 \pm 8.9 \mathrm{pg} / \mathrm{ml}$ vs. $7.57 \pm 3.73 \mathrm{pg} / \mathrm{ml}, p=$ 0.093) (Figure 4).

When comparing the AR patients depending on the presence of nasal polyposis, some differences in the serum levels of the cytokines occurred. In patients with nasal polyposis we found a weak trend towards significantly lower levels of IL-4 $(4.20 \pm 1.6 \mathrm{pg} / \mathrm{ml})$ and non-significantly lower IL-5 levels $(5.77 \pm 1.3 \mathrm{pg} / \mathrm{ml})$ compared to the patients without polyposis ( $35.00 \pm 21.59 \mathrm{pg} / \mathrm{ml}, p=0.165$ for IL-4 and $8.8 \pm 3.66 \mathrm{pg} / \mathrm{ml}, p=0.459$ for IL-5) (Figure 5). On the other hand, patients with nasal polyposis had higher IL-13 in the serum compared to the patients without polyposis ( $31.43 \pm 17.68 \mathrm{pg} / \mathrm{ml}$ vs. $13.69 \pm 4.55 \mathrm{pg} / \mathrm{ml}$, $p=0.493)$, with no levels of significance.

\section{Discussion}

The interaction between environmental allergens and corresponding lgE antibody is an essential precondition for allergic airway reactions and a major pathogenetic mechanism triggering the symptoms of allergic rhinitis and bronchial asthma. First immunopathogenetic response required for onset of these diseases is increased

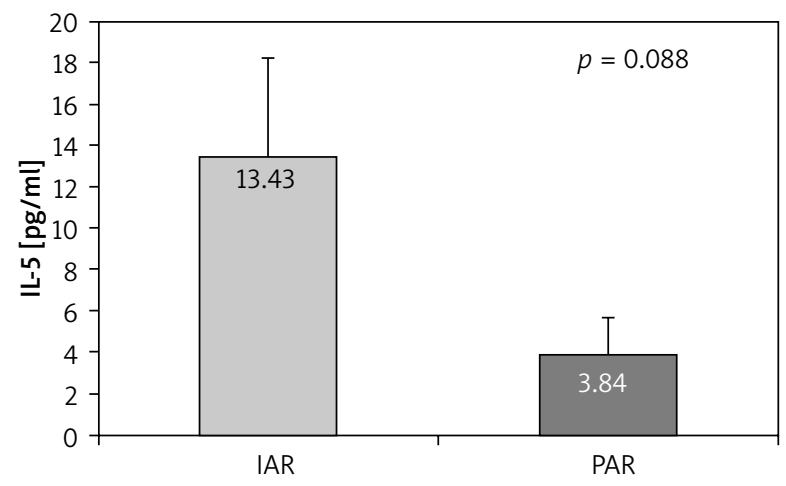

Figure 2. IL-5 in the lavage of patients with IAR and PAR

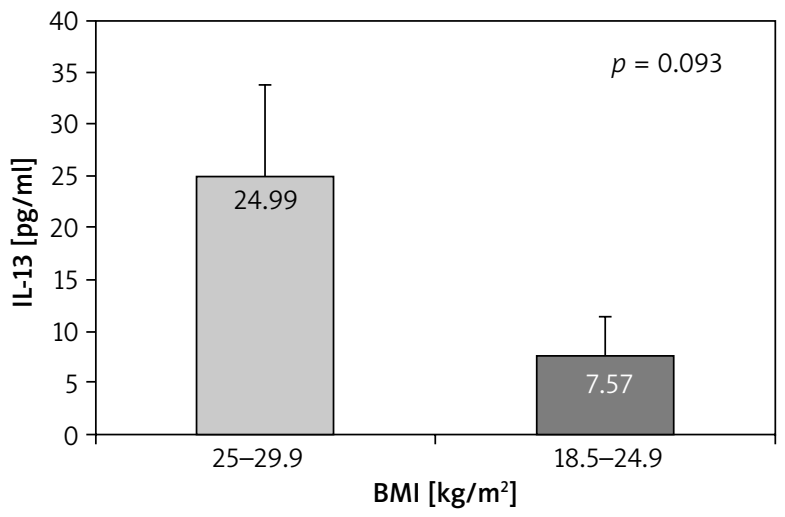

Figure 4. IL-13 serum levels in AR patients according to BMI 


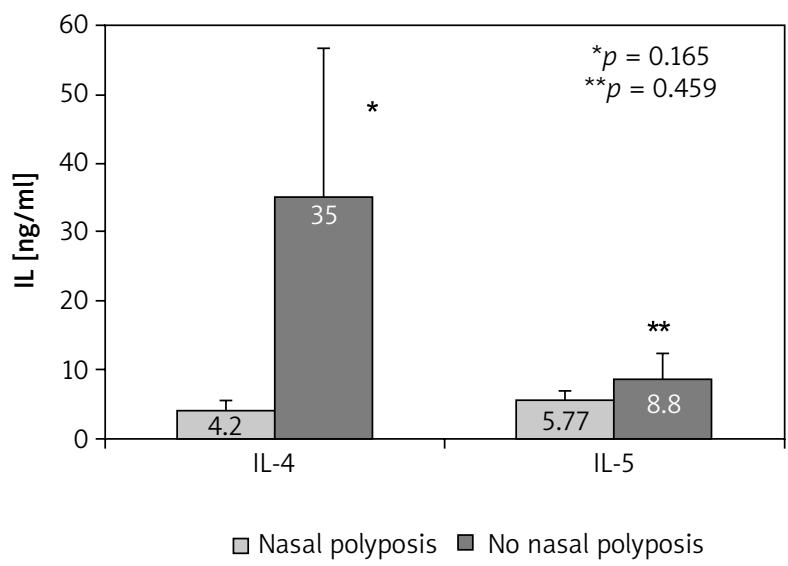

Figure 5. IL-4 and IL-5 serum levels in AR patients with and without nasal polyposis

production of IgE antibodies against environmental-specific allergens such as house dust and pollen. A number of studies suggest that the production of IgE can be regulated by antigen specific helper and suppressor T cells and isotype-specific factors showing IgE affinity. Another way of regulating IgE based on the phenotypic and functional activities of various T-cell subgroups is by using cytokines, produced by Th-2 cells, which have a fundamental role in inducing and enhancing production of specific IgE antibodies by B lymphocytes. This explains why Th-2helper cells are predominant in allergic conditions [8].

Studies have shown that innate immune response cells, such as macrophages and dendritic cells, which carry out first and nonspecific contact with foreign antigens, can induce Th2 responses. It is believed that they can influence production of IL-4 or immunoglobulin-E and sub-class switching of lymphocytes in the initial phases of AP in rats [9].

In a study, Ohashi et al. have found that serum total IgE levels did not differ between the three groups of healthy participants, atopic patients at the time of clinical exposure, and atopic patients out of the clinical manifestation period, in an experiment to observe the spontaneous IgE synthesis [10].

According to Finkelman et al., the level of specific IgE is more important in allergic diseases than the IgEtotal level. In addition, IL-4 plays a fundamental role in inducing and increasing the generation of both primary polyclonal and secondary IgE specific B lymphocyte responses [11].

In their experiments with nasal lavage, Lee et al. have been able to prove significantly higher levels not only of specific IgE molecules, but also the general IgE, compared with healthy controls [12].

In our study we fail to find differences in the total lgE in the serum and lavage between controls and subjects with AR. Despite the lack of statistically significant dif- ferences, in approximately $25 \%$ of our controls without allergic pathology we determined lower levels of the antibody.

Already in 1988 Prete et al. detected elevated IL-4 levels and suggested that this molecule plays a critical role in switching B-lymphocytes to IgE production [13].This critical role of IL-4 in isotype switching is corroborated by the observation that mice with IL-4 germline gene disruption failed to generate Th-2 cells in vivo and produce IgE antibodies [14].

Ohashi et al. in their efforts to demonstrate the involvement of IL-4 in modulating the process of atopic response in patients with $A R$, as well as the treatment response, have measured the serum cytokine concentration in patients with clinically manifested AR before and after subcutaneous hyposensitization therapy [15]. The same authors have reported significantly lower serum IL-4 levels in the non-atopic group compared to those with atopy. This constellation is confirmed by other authors showing significantly reduced IL-4 and IgE levels as a result of immunotherapy, unlike the group that had undergone antihistamine treatment, in combination with or without a local corticosteroid. According to the authors, a decreasing serum concentration of specific IgE does not correlate with the severity of clinical manifestation of the symptoms $[16,17]$.

In their study, Krouse et al. have also found elevated IL-4 serum levels in AR patients, in combination with $\mathrm{IL}-1 \beta$ and IL-10, in healthy subjects. By comparing concentrations with sleep quality and clinical manifestation of AR symptoms, they have determined these cytokines as "pro-allergenic" molecules. Higher levels of pro-allergenic molecules have resulted in sleep disturbances detected in polysomnographic records [18].

Our results show higher IL-4 levels in the serum of patients with AR compared to controls, as well as increased concentrations of the cytokine in the lavage of patients with the intermittent form compared to those with the persistent form. These data indicate enhanced inflammatory activity in the pollen induced form. In addition overweight patients had higher IL-4 levels in the blood compared to the those with normal weight and underweight.

In a number of studies, elevated levels of IL-4 and IL-13 in nasal secretions have been demonstrated in patients with allergic rhinitis. For example, Scavuzzo et al. have been able to measure significantly elevated IL-4 molecules in AR patients in comparison with healthy subjects [19]. Ciprandi et al. have also reported a positive significant correlation between IL-4 and IL-5 concentration in patients with AR and inflammatory eosinophils in the lavage fluid [20].

In the scientific literature, results demonstrating no elevation in IL-4, IL-6, IL-8, leukotriene C4, B4, and prostaglandin E2 levels in atopic rhinitis patients are found [21], and others where after 2 weeks of intranasal corticoste- 
roid administration, no change in cytokine activity was detected but only in the number of Eo cells [22].

We did not find any significant difference in the serum levels of IL- 5 in patients and controls, but still patients with IAR had higher levels of this cytokine in the lavage compared to those with PAR.

The control individuals in our group had lower IL-13 levels in the blood although with no significant differences.

When comparing concentrations of certain cytokine molecules in healthy people and patients with allergic and viral rhinitis, Klemens et al. have found IL-13 in a very small percentage of clinical cases. Another interesting finding is the increased levels of IL-5 in patients with viral-induced rhinitis. IL-4 concentration has been increased in the two clinical groups, especially in inflammatory ones [23].

Marra et al. have demonstrated elevated levels of IL-5 in induced sputum in patients only with allergic rhinitis and those only suffering from bronchial asthma. These findings confirm the presence of a close connection between the upper and lower respiratory tract and the same or close immunological reactivity of nasal cavity and bronchial lining [24].

Benson et al. have failed to detect a statistically significant difference in IL-5 concentration in off-season nasal lavage of controls and AR patients, but have found slightly increased levels in high pollen concentration seasons as well as a decrease in IL-5 values in nasal secrets after topical application of Budesonide spray [25]. However, it is believed that IL-5 is one of the key cytokines responsible for bone marrow eosinopoiesis as well as the survival of eosinophils in tissues and proving its presence in inflammatory tissues in patients with mild or even asymptomatic bronchial asthma, which suggests that this cytokine can be considered as a good marker for inflammation of airways in atopic patients [26].

\section{Conclusions}

Regardless of the obvious differences of the concentrations of the cytokines studied in our groups, oftentimes no significant difference is observed. We suggest this might be due to the small number of the individuals in all groups. More studies should be conducted in order to show the role of IL-4, $-5,-13$, and IgE in the pathogenesis and severity of the disease.

\section{Acknowledgments}

The study was conducted in the Department of Otorhinolaryngology and Ophthalmology and the Department of Chemistry and Biochemistry, Medical Faculty, Trakia University, Stara Zagora, Bulgaria, as well as the University Hospital, Stara Zagora, Bulgaria.

\section{Conflict of interest}

The authors declare no conflict of interest.

\section{References}

1. International Consensus Report on the diagnosis and management of rhinitis. International Rhinitis Management Working Group. Allergy 1994; 49 (19 Suppl): 1-34.

2. King HC, Mabry RL, Gordon BR, et al. Allergy in ENT Practice: The Basic Guide. $7^{\text {th }}$ ed. Thieme, New York 2005.

3. Galli SJ, Tsai M. IgE and mast cells in allergic disease. Nat Med 2012; 18: 693-704.

4. Rondón C, Campo P, Togias A, et al. Local allergic rhinitis: concept, pathophysiology, and management. J Allergy Clin Immunol 2012; 129: 1460-7.

5. Wheatly LM, Togias A. Allergic rhinitis. N Engl J Med 2015; 372: 456-63.

6. Howarth PW. Allergic and non allergic rhinitis. In: Middleton's Allergy Principles and Practice. Adkinson F Jr, Yunginger JW, Busse WW, et al. (eds). Mosby, Pennsylvania 2003; 1391-414.

7. Deraz TE. Immunopathogenesis of allergic rhinitis. Egypt J Pediatr Allergy Immunol 2010; 8: 3-7.

8. Ricci M, Rossi O, Bertoni M, Matucci A. The importance of Th-2-like cells in the pathogenesis of airway allergic inflammation. Clin Esp Allergy 1993; 23: 360-9.

9. Gergen PJ, Arbes SJ Jr, Calatroni A, et al. Total IgE levels and asthma prevalence in the US population: results from the National Health and Nutrition Examination Survey 20052006. J Allergy Clin Immunol 2009; 124: 447-53.

10. Ohashi Y, Nakai Y, Tanaka A, et al. Allergen-induced synthesis of interleukin-5, but not of IgE, is a key mechanism linked to symptomatic episodes of seasonal allergic rhinitis in sensitized individuals. Scand J Immunol 1998; 47: 596-602.

11. Finkelman F, Holmes J, Katona $\mathrm{H}$, et al. Lymphokine control of in vivo immunoglobulin isolype selection. Annu Rev Immunol 1990; 8: 303-33.

12. Lee KS, Yu J, Shim D, et al. Local immune responses in children and adults with allergic and nonallergic rhinitis. PLoS One 2016; 11: e0156979..

13. Prete GD, Maggi E. Parronchi P, et al. IL-4 is an essential factor for the IgE synthesis induced in vitro by human T cell clones and their supernalants. J Immunol 1988; 140: 4193-8.

14. Kopf M, Le Gros G, Bachman M, et al. Disruption of the murine IL-4 gene blocks Th2 cytokine responses. Nature 1993; 362: 245-8.

15. Ohashi Y, Nakai Y, Okamoto H, et al. Serum level of interleukin-4 in patients with perennial allergic rhinitis during allergen-specific immunotherapy. Scand I Immunol 1996; 43: 680-6.

16. Matsumoto T, Miike T, Yamaguchi K, et al. Serum levels of soluble IL-2 receptor, IL-4 and IgE-binding factors in childhood allergic diseases. Clin Exp Immunol 1991; 85: 288-92.

17. Reddy M, Weissman A, Mazza D, et al. Circulating elevated levels of soluble CD23. interleukin-4. and CD20CD23 lymphocytes in atopic subjects with elevated serum IgE concentrations. Ann Allergy 1992; 69: 131-4.

18. Krouse H, Davis J, Krouse J. Immune mediators in allergic rhinitis and sleep. Otolaryngol Head Neck Surg 2002; 126: 607-13.

19. Scavuzzo MC, Rocchi V, Fattori B, et al. Cytokine secretion in nasal mucus of normal subjects and patients with allergic rhinitis. Biomed Pharmacother 2003; 57: 366-71. 
20. Ciprandi G, Vizzaccaro A, Cirillo I, et al. Nasal eosinophils display the best correlation with symptoms, pulmonary function and inflammation in allergic rhinitis. Int Arch Allergy Immunol 2005; 136: 266-72.

21. Linden M, Greiff L, Andersson M, et al. Nasal cytokines in common cold and allergic rhinitis. Clin Exp Allergy 1995; 25: 166-72.

22. Sulakvelidze I, Conway M, Evans S, et al. Clinical and nasal irrigation fluid findings in perennial allergic rhinitis. Am J Rhinol 1997; 11: 435-41.

23. Klemens C, Rasp G, Jund F, et al. Mediators and cytokines in allergic and viral-triggered rhinitis. Allergy Asthma Proc 2007; 28: 434-41.

24. Marra S, Borges R, Alves R, et al. Spirometric parameters and levels of interferon gamma and IL-5 in induced sputum from patients with allergic rhinitis or asthma. Am J Rhinol Allergy 2011; 25: e196-9.

25. Benson M, Strannegaerd I, Wennergren G, Strannegaerd O. Interleukin-5 and interleukin-8 in relation to eosinophils and neutrophils in nasal uids from school children with seasonal allergic rhinitis. Pediatr Allergy Immunol 1999; 10: 178-85.

26. Leckie MJ. Anti-interleukin-5 monoclonal antibodies: preclinical and clinical evidence in asthma models. Am J Respir Med 2003; 2: 245-59. 